\title{
«Ärzte sind es nicht gewohnt, Hilfe in Anspruch zu nehmen»
}

\author{
Ärztinnen und Ärzte in Krisensituationen können dank ReMed auf Hilfe zählen. Nach \\ einer positiven Pilotphase ist das Unterstützungsnetzwerk nun ab sofort in der ge- \\ samten Schweiz aktiv. Was ReMed genau ist, wie es funktioniert und an wen es sich \\ richtet, erläutert Dr. med. Michael Peltenburg, Mit-Initiant und Programmleiter von \\ ReMed, im nachfolgenden Interview.
}

Interview:

Jacqueline Wettstein

Leiterin Kommunikation FMH

Korrespondenz:

Dr. med. Michael Peltenburg Facharzt für Allgemeinmedizin FMH CH-8340 Hinwil

michael.peltenburg@hin.ch

\section{Was ist ReMed?}

Michael Peltenburg: Wir Ärztinnen und Ärzte haben einen spannenden, aber auch belastenden Beruf. Unsere Arbeit ist anspruchsvoll, weil es um Menschen und deren Leben geht. Wenn ein Arzt in einer Krise steckt, kann das Auswirkungen auf die Qualität seiner Arbeit haben. Deshalb bietet ReMed genau in solchen Situationen Unterstützung: Wir begleiten betroffene Ärztinnen und Ärzte und zeigen in scheinbar ausweglosen Situationen neue Möglichkeiten auf.

\section{Wie funktioniert ReMed?}

Der erste Kontakt bei ReMed läuft über einen der vier Ärztinnen und Ärzte des Leitungsausschusses. Der jeweilige Berater bespricht zusammen mit dem ratsuchenden Arzt die weitere Vorgehensweise: Was ist der Grund für die Kontaktaufnahme? In welcher Form soll die Unterstützung erfolgen? ReMed ist somit vor allem eine Anlaufstelle, die eine Krisenintervention, Mentoring, Coaching und Assessment anbietet. Je nachdem, was das Problem ist, vermittelt der Berater geeignete Fachpersonen - zum Beispiel Psychiaterinnen und Psychologen - oder spezifische Institutionen.

Wer kann sich bei ReMed melden?

ReMed richtet sich an Ärztinnen und Ärzte, die sich in einer Krise befinden. Und auch Angehörige und Mitarbeitende von Ärzten können Kontakt mit ReMed aufnehmen. ReMed meldet sich dann innerhalb von 72 Stunden, meist sogar rascher. Unsere Erfahrung zeigt, dass sich die Hilfesuchenden häufig erst sehr spät an uns wenden. Deshalb raten wir, möglichst frühzeitig, bevor die Krise auf dem Höhepunkt ist, mit ReMed Kontakt aufzunehmen.

\section{Wer meldet sich und mit welchen Beschwerden?}

Ärztinnen und Ärzte wenden sich mit ganz unterschiedlichen Problemen an uns. Das kann zum Beispiel ein Konflikt mit dem Vorgesetzten sein oder auch eine fortwährende berufliche Überlastung. Selbstverständlich ist ReMed auch da, wenn es um

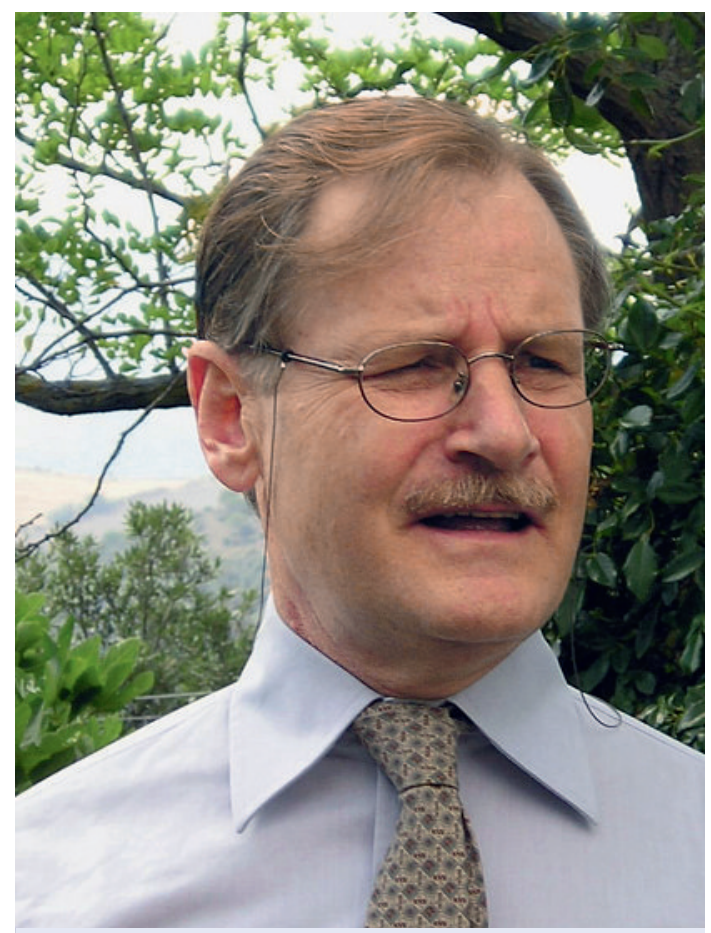

Dr. med. Michael Peltenburg, Mit-Initiant und Programmleiter von ReMed.

persönliche Schwierigkeiten von Ärztinnen und Ärzten geht - etwa um anhaltende Konflikte zu Hause oder um eine Scheidung.

Was kann ReMed bewirken?

Ärztinnen und Ärzte stehen täglich für ihre Patienten im Einsatz. Doch wenn es um sie selbst geht, sind sie es nicht gewohnt, Hilfe in Anspruch zu nehmen. Häufig fällt es ihnen schwer, sich einzugestehen, dass sie Unterstützung brauchen. Bei ReMed treffen Ratsuchende auf Ärzte als Berater. Diese wissen, wovon sie sprechen, und kennen die Schwierigkeiten aus eigener Erfahrung. Wir möchten dazu beitragen, dass Ärzte gesund werden und bleiben. Und dass sie weiterhin mit Freude ihrem Beruf nachgehen und damit 
eine qualitativ hochstehende und sichere medizinische Versorgung der Bevölkerung gewährleisten.

ReMed will ja auch präventiv wirken - wie geht das? Die Ärzteschaft kümmert sich häufig viel zu wenig um die eigene Gesundheit. Mit ReMed wollen wir unseren Berufskollegen genau dies bewusst machen: Die eigene Gesundheit ist wichtig, und wir müssen Sorge zu ihr tragen.

Wer steht hinter ReMed?

Trägerin ist die FMH: Als wir das Projekt dem Zentralvorstand vorgestellt haben, waren die Rückmeldungen sofort positiv. Wir führen das Projekt zusammen mit der Abteilung Daten, Demographie und Qualität DDQ der FMH durch. Auch wenn die FMH die finanziellen Mittel für ReMed zur Verfügung stellt, hat sie zu keinem Zeitpunkt Einblick in die Personen- und Betreuungsakten. ReMed ist an das ärztliche Berufsgeheimnis gebunden.

\section{Die FMH hat zu keinem Zeitpunkt Einblick in die Personen- und \\ Betreuungsakten}

Die Pilotphase ist abgeschlossen, und ab Herbst 2010 wird ReMed schweizweit angeboten. Was sind nun die nächsten Schritte?

Seit der Projektlancierung 2007 ist ReMed fast 80 Mal aktiv geworden. Das zeigt, dass die Ärztinnen und Ärzte Vertrauen zu ReMed haben. Nun geht es natür- lich darum, ReMed noch bekannter zu machen. ReMed wird jetzt offiziell auf die gesamte Schweiz ausgeweitet. Dazu haben wir eine ganze Reihe von Kommunikationsmassnahmen geplant -z.B. Flyer oder Kleinplakate für Spitäler. Ärzte müssen wissen, dass sie sich in Krisen an ReMed wenden können.

\section{ReMed - für Ärzte in Krisensituationen} ReMed berät und begleitet Ärztinnen und Ärzte, die sich in einer Krise befinden. Das kann ein Konflikt am Arbeitsplatz sein, aber auch eine persönliche Krise, wie etwa eine Scheidung. Das Unterstützungsnetzwerk meldet sich innerhalb von 72 Stunden nach der ersten Kontaktaufnahme. Anschliessend begleitet ReMed die Ratsuchenden und zeigt Handlungsmöglichkeiten auf. ReMed ist an das ärztliche Berufsgeheimnis gebunden.

\section{Kontakt}

Befinden Sie sich in einer schwierigen Situation und suchen Rat? Oder kennen Sie einen Arzt in Ihrem Umfeld, der Unterstützung benötigt? Dann melden Sie sich bei ReMed über die 24-Stunden-Hotline: 0800073633 oder über help@swiss-remed.ch.

\section{Informationen}

Möchten Sie mehr wissen und auch Ihre Kolleginnen und Kollegen auf ReMed aufmerksam machen? Flyer und Kleinplakate auf Deutsch oder Französisch können Sie kostenlos über info@swiss-remed.ch anfordern. Zusätzliche Informationen können Sie auch finden unter www.swiss-remed.ch.

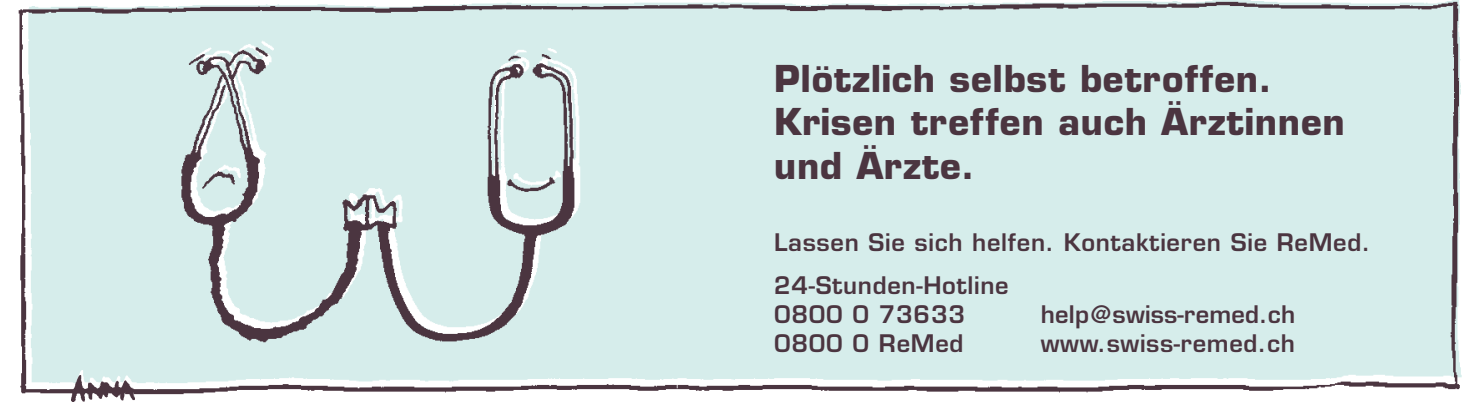

